\section{Mini-Review}

Correspondence

Stefanie Pöggeler

spoegge@gwdg.de

\title{
Carbonic anhydrases in fungi
}

\author{
Skander Elleuche and Stefanie Pöggeler
}

Institute of Microbiology and Genetics, Department of Genetics of Eukaryotic Microorganisms, Georg-August University, Göttingen, Germany

\begin{abstract}
Carbonic anhydrases (CAs) are metalloenzymes that catalyse the interconversion of carbon dioxide and bicarbonate with high efficiency. This reaction is fundamental to biological processes such as respiration, photosynthesis, $\mathrm{pH}$ homeostasis, $\mathrm{CO}_{2}$ transport and electrolyte secretion. CAs are distributed among all three domains of life, and are currently divided into five evolutionarily unrelated classes $(\alpha, \beta, \gamma, \delta$ and $\zeta)$. Fungal CAs have only recently been identified and characterized in detail. While Saccharomyces cerevisiae and Candida albicans each have only one $\beta$-CA, multiple copies of $\beta$-CA- and $\alpha$-CA-encoding genes are found in other fungi.

Recent work demonstrates that CAs play an important role in the $\mathrm{CO}_{2}$-sensing system of fungal pathogens and in the regulation of sexual development. This review focuses on CA functions in $S$. cerevisiae, the fungal pathogens C. albicans and Cryptococcus neoformans, and the filamentous ascomycete Sordaria macrospora.
\end{abstract}

\section{Introduction}

Carbon dioxide $\left(\mathrm{CO}_{2}\right)$ is a small molecule that is among the most important gases for all living organisms. It is a waste product of respiration, but it is also a nutrient, a sensing factor and a major component of the carbon cycle. Despite its ubiquitous importance, $\mathrm{CO}_{2}$ comprises only $0.033 \%$ of the atmospheric gases, although it is found at higher levels in several tissues. Plants, algae and cyanobacteria are able to fix $\mathrm{CO}_{2}$ during photosynthesis, while animals and micro-organisms produce and return it to the atmosphere.

In nature, the concentration of $\mathrm{CO}_{2}$ is balanced by an interconversion to bicarbonate $\left(\mathrm{HCO}_{3}^{-}\right)$through the spontaneous reaction $\mathrm{CO}_{2}+\mathrm{H}_{2} \mathrm{O} \leftrightarrow \mathrm{HCO}_{3}^{-}+\mathrm{H}^{+}$. Bicarbonate is also an important biological substrate, and since the average amount of $\mathrm{HCO}_{3}^{-}$required by organisms is much greater than the amount produced spontaneously from $\mathrm{CO}_{2}$, bicarbonate production requires finely tuned regulation (Jones, 2008). For this reason, a highly diverse family of enzymes has evolved that are able to accelerate the interconversion reaction by up to 10000 -fold (Wistrand, 1981). Members of this protein family, called carbonic anhydrases (CAs), are able to catalyse the reversible hydration of $\mathrm{CO}_{2}$ to give $\mathrm{HCO}_{3}^{-}$and a proton (Supuran, 2008). The production of $\mathrm{H}^{+}$results in a $\mathrm{pH}$ decrease, so CAs are also involved in $\mathrm{pH}$ homeostasis. In addition, CAs are involved in biosynthesis or detoxification pathways that use $\mathrm{HCO}_{3}^{-}$as a co-factor or as a co-substrate, such as fattyacid or arginine biosynthesis, the cAMP pathway and cyanate degradation (Aguilera et al., 2005b; Anderson et al., 1990; Bahn \& Mühlschlegel, 2006).

CAs have evolved in all three domains of life and are currently divided into five, evolutionarily unrelated classes $(\alpha, \beta, \gamma, \delta$ and $\zeta)$ that independently arose from different precursors during convergent evolution. The best-studied group is the $\alpha$-class of mammals, prokaryotes, plants and fungi. The $\beta$-class has been identified in plants, bacteria and fungi but not mammals, while $\gamma$-CAs, which have strikingly different sequence features, are predominantly found in archaea (Supuran, 2008; Tripp et al., 2001). The $\beta$-class can be further subdivided into three subclasses. The plant-like $\beta$-CAs and the cab-like $\beta$-CAs, named after the carbonic anhydrase $\beta$ (CAB) from the archaeon Methanobacterium thermoautotrophicum, were first described by Kimber \& Pai (2000). The third subclass of $\beta$-CAs, which is designated the $\varepsilon$-class, was identified in the chemolithoautotrophic bacterium Halothiobacillus neapolitanus (Sawaya et al., 2006). Recently, two more CA classes, the $\delta$ - and $\zeta$-classes, have been described. So far, these have been found only in marine diatoms (Xu et al., 2008). Although the primary structures of the various classes of CAs are strikingly different, the metal- (in most cases a zinc atom) coordinating sites are remarkably similar at the structural level. The number of subunits also varies greatly among the different CA classes. Nonetheless, all CA enzymes catalyse the same chemical reaction (Supuran, 2008; Tripp et al., 2001).

At least $16 \alpha$-CAs or carbonic-anhydrase-related proteins (CARPs) are encoded in mammals (Supuran, 2008, and references therein). These isoenzymes are tissue or organ specific in their expression and localize to different subcellular compartments. The cytosolic isozymes CA I and CA II were first characterized in human and bovine blood cells, where they play a significant role in gas exchange, while the distribution of CA III is restricted to the cytosol of skeletal muscles and adipocytes. The remaining cytosolic CA VII and the CARPs CA VIII, X 
and XI were demonstrated to be located in the central nervous system and CA XIII was detected in the kidney, liver and brain. Mitochondrial CAs are exclusively localized in the liver (CA VA) or distributed in heart and skeletal muscles, kidney, pancreas, spinal cord and gastrointestinal tract $(\mathrm{CA} \mathrm{VB})$. Five isoforms are associated with the membrane of cells in the kidney (CA IV, CA XIV and CA $\mathrm{XV}$ ), heart muscles (CA IV), brain (CA IV and CA XIV), pancreas (CA IV), liver (CA XIV) and eye (CA XII). CA IX is a transmembrane enzyme with significant scientific relevance, because of its clinical application as a tumour marker. CA VI is a secreted protein, present in salivary and mammary glands (Supuran, 2008). Phylogenetic analysis has revealed that all human CA isoenzymes clearly originated from a common ancestor.

Since CAs are a ubiquitous group of enzymes, the conservation of their physiological roles among organisms is worth investigating, because it suggests a complex evolutionary history. Genome sequencing projects have identified CAs in all organisms analysed. To the best of our knowledge, only Symbiobacterium thermophilum, a syntrophic bacterium that effectively grows on $\mathrm{CO}_{2}$ generated by other bacteria, has lost its CA genes (Nishida et al., 2009).

\section{Fungi possess $\alpha$ - and $\beta$-CAs}

Fungi react to different stimuli, such as temperature and nutrients. Gases can also serve as signalling molecules (Bahn et al., 2007). A prominent example of a gas-driven process is the switching of Candida albicans between yeast and filamentous growth in response to varying $\mathrm{CO}_{2}$ levels. This process was demonstrated to be controlled by a fungal adenylyl cyclase and a $\beta$-CA (Klengel et al., 2005).

All known fungal CAs belong either to the $\alpha$ - or to the $\beta$ class (Elleuche \& Pöggeler, 2009a). To date, members of the other three groups have not been identified in fungal genomes. Fungal CA-encoding genes, like the CA multigene families in mammals, algae and plants, have diversified extensively during fungal evolution. The genomes of most filamentous ascomycetes contain three isoforms of $\beta$-class CAs and at least one $\alpha$-class CA, whereas only $\beta$-class CAs have been identified in hemiascomycetous yeasts (Elleuche \& Pöggeler, 2009a).
Within the basidiomycetes, only Cryptococcus neoformans encodes two $\beta$-CAs, while the Coprinopsis cinerea, Laccaria bicolor, Malassezia globosa and Ustilago maydis genomes all contain single $\beta$-CA genes (Elleuche \& Pöggeler, 2009a). Interestingly, eight genes encoding putative $\alpha$-CAs can be identified in the genome of L. bicolor (S. Pöggeler, unpublished).

$\beta$-CAs have been functionally characterized in only a few fungal species (Table 1). The NCE103 gene encodes a plantlike $\beta$-CA in the hemiascomycetous yeasts Candida albicans, Candida glabrata and Saccharomyces cerevisiae, whereas two closely related isozymes, Can1 and Can2, have been described in the basidiomycete Cryptococcus neoformans (Bahn et al., 2005; Götz et al., 1999; Innocenti et al., 2009; Klengel et al., 2005). Interestingly, an additional putative cab-like $\beta$-CA has been identified in the pathogenic yeast C. albicans (XP_715817) and in Pichia stipitis (XP_001383682.1) (Elleuche \& Pöggeler, 2009a). Recently, a multigene family of $\beta$-CAs was shown to influence the sexual development of the filamentous ascomycete Sordaria macrospora (Elleuche \& Pöggeler, 2009b). CA activity from secreted CA isoforms has also been detected in various Penicillium isolates, and this activity is believed to be involved in limestone dissolution (Li et al., 2009).

\section{The CA Nce103 of Saccharomyces cerevisiae}

The first fungal CA was discovered in 1996, in the yeast $S$. cerevisiae. Initially, it was identified as a component of a non-classical protein export pathway and designated Nce103 (Cleves et al., 1996). Subsequent sequence alignments of the predicted amino acid residues assigned Nce103 to the $\beta$-class of CAs (Götz et al., 1999). A deletion mutant of NCE103 is unable to grow under ambient air conditions $\left(0.033 \% \mathrm{CO}_{2}\right)$. Since the lethality of the $n c e 103 \Delta$ mutation can be complemented by high $\mathrm{CO}_{2}$ concentration $\left(5 \% \mathrm{CO}_{2}\right)$, the phenotype was defined as high- $\mathrm{CO}_{2}$-requiring (HCR). However, the defect of the

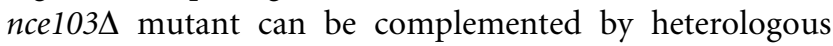
expression of CAs from plants [ $\beta$-CA from Medicago sativa, tobacco chloroplast $\beta$-CA (Götz et al., 1999; Slaymaker et al., 2002)], mammals [human $\alpha$-CAII (Clark et al.,

Table 1. Functionally characterized $\beta$-CAs in fungi

\begin{tabular}{|lll|}
\hline Species & Protein & \multicolumn{1}{c|}{ Reference } \\
\hline Candida albicans & Nce103 & Klengel et al. $(2005)$ \\
Candida glabrata & Nce103 & Innocenti et al. $(2009)$ \\
Cryptococcus neoformans & Can1 & Bahn et al. $(2005)$ \\
& Can2 & Bahn et al. (2005) \\
Saccharomyces cerevisiae & Nce103 & Cleves et al. (1996); Götz et al. (1999) \\
Sordaria macrospora & CAS1 & Elleuche \& Pöggeler (2009b) \\
& CAS2 & Elleuche \& Pöggeler (2009b) \\
& CAS3 & Elleuche \& Pöggeler (2009b) \\
\hline
\end{tabular}


2004)], bacteria [ $\beta$-CA can from Escherichia coli (Cronk et al., 2001)] or C. neoformans [ $\beta$-CA Can1 and Can2 (Bahn et al., 2005)], indicating that non-specific CA activity is sufficient to ensure an efficient supply of $\mathrm{HCO}_{3}^{-}$for yeast cells.

In addition, it has been shown that NCE103 is transcriptionally upregulated under ambient air conditions $(0.033 \%$ $\mathrm{CO}_{2}$ ), and the detectable CA activity corresponds to an increase in NCE103 mRNA accumulation under inducing, low- $\mathrm{CO}_{2}$, conditions (Amoroso et al., 2005). High levels of $\mathrm{CO}_{2}$ result in the downregulation of NCE103 transcript (Aguilera et al., 2005a). In agreement with these results, no $\mathrm{CA}$ activity is detectable in cells grown at high (5\%) $\mathrm{CO}_{2}$ levels (Amoroso et al., 2005).

The HCR phenotype of the S. cerevisiae nce103A mutant indicates the importance of $\mathrm{Ncel} 103$ in $\mathrm{CO}_{2} / \mathrm{HCO}_{3}^{-}$ homeostasis and suggests that $S$. cerevisiae $\mathrm{CA}$ may participate in supplying intracellular $\mathrm{HCO}_{3}^{-}$for anaplerosis or for the regulation of the intracellular $\mathrm{pH}$ (Amoroso et al., 2005). Aguilera et al. (2005b) identified several $\mathrm{HCO}_{3}^{-}$dependent carboxylation reactions that are impaired in the $n c e 103 \Delta$ mutant strain. To test the nutritional requirements of the nce $103 \Delta$ mutant under non- $\mathrm{CO}_{2}$-enriched conditions, the mutant was complemented by adding L-aspartate, Larginine, uracil or fatty acids to the medium. The results demonstrated that the main physiological role of the $S$. cerevisiae CA is the production of bicarbonate for $\mathrm{HCO}_{3}^{-}$dependent metabolic carboxylation reactions catalysed by pyruvate carboxylase, acetyl-CoA carboxylase and carbamoyl-phosphate synthetase under low $\mathrm{CO}_{2}$ concentrations (Aguilera et al., 2005b).

\section{CAs of the fungal pathogens Candida albicans and Cryptococcus neoformans}

The role of CAs in development and virulence has been investigated in the pathogenic fungi Candida albicans and Cryptococcus neoformans (Bahn \& Mühlschlegel, 2006; Mogensen \& Mühlschlegel, 2008), which are both exposed to drastic environmental changes during their infectious life cycles. In its natural habitat, C. neoformans grows as a saprophyte at $0.033 \% \mathrm{CO}_{2}$, but when it infects the human lung, it must adapt to levels as high as $5 \% \mathrm{CO}_{2}$. Similarly, C. albicans can survive low levels of $\mathrm{CO}_{2}$ such as on the skin, but it is also able to infect the bloodstream in immunocompromised patients, where the $\mathrm{CO}_{2}$ concentration is $\sim 5 \%$. Elevated $\mathrm{CO}_{2}$ concentrations promote a prominent switch from yeast to hyphal growth in the hemiascomycetous yeast C. albicans and the induction of capsule biosynthesis in the basidiomycete (Bahn et al., 2005; Klengel et al., 2005).

The CA-encoding genes CAN1 and CAN2 of C. neoformans, and NCE103 of C. albicans, have been characterized. All these genes encode $\beta$-class CAs and are able to rescue CA deletion strains of E. coli or S. cerevisiae (Bahn et al., 2005; Mogensen et al., 2006). The crystal structure of $C$. neoformans Can2 revealed that the enzyme carries a unique $\mathrm{N}$-terminal extension that can interact with the active site entrance of the dimer, so the N-terminus was hypothesized to be an internal regulator or an interaction site for a different protein (Schlicker et al., 2009). Deletion analysis of the CA-encoding genes in C. neoformans and C. albicans revealed that CAN2 and NCE103 are essential for survival under ambient air, but dispensable for in vivo proliferation and virulence at the high $\mathrm{CO}_{2}$ levels found in the host (Bahn et al., 2005; Klengel et al., 2005). In C. neoformans, the in vitro growth defects under ambient air were largely attributable to defective fatty acid synthesis. The $C$. neoformans CAN2 mutant had physiological impairments in addition to the virulence defect. The mating ability of $C$. neoformans wild-type is inhibited under high- $\mathrm{CO}_{2}$ conditions, but the can $2 \Delta$ strain had lost this regulation and initiated mating independently of the $\mathrm{CO}_{2}$ level. This showed that $\mathrm{HCO}_{3}^{-}$, as the conversion product of $\mathrm{CO}_{2}$, is the key molecule for mating inhibition. Furthermore, the transcription of pheromone genes no longer decreases at elevated $\mathrm{CO}_{2}$ conditions in the can $2 \Delta$ strain (Bahn et al., 2005).

Interestingly, CAs of both these fungal pathogens are involved in $\mathrm{CO}_{2}$ sensing and virulence. In both fungi, adenylyl cyclase (AC) was shown to transmit the $\mathrm{CO}_{2}$ signal in a CA-dependent manner (Bahn et al., 2005; Klengel et al., 2005; Mogensen et al., 2006). Like the mammalian and bacterial soluble ACs (Chen et al., 2000), the catalytic domains of C. albicans and C. neoformans ACs were shown to be activated by bicarbonate in vitro (Klengel et al., 2005; Mogensen et al., 2006).

\section{Sordaria macrospora encodes four carbonic anhydrases}

The filamentous ascomycete $S$. macrospora is a close relative of the well-known red bread mould Neurospora crassa, and is used as a model system for investigating processes of sexual development (Kück et al., 2009; Nowrousian et al., 2004; Pöggeler et al., 2006). The role of $\mathrm{HCO}_{3}^{-}$metabolism during sexual reproduction has been investigated by characterizing three $\beta$-CA genes (Elleuche \& Pöggeler, 2009b). A total of four CA-encoding genes, cas 1 , cas 2 , cas 3 and cas4, have been identified in the $S$. macrospora genome. CAS1 and CAS2 are closely related proteins that belong to the plant-like subgroup of $\beta$-CAs, while cas 3 encodes a cab-type $\beta$-CA and cas 4 an $\alpha$-CA. Based on the similarity of cas 1 and cas2, these genes are proposed to be the result of an ancient gene duplication event (Elleuche \& Pöggeler, 2009a). In contrast to the widely distributed plant-like $\beta$-CAs in fungi, cab-type $\beta$-CA genes seem to be restricted to the ascomycete phylum. Filamentous ascomycetes of the order Hypocreales exhibit multiple cab-like $\beta$-CAs, but only a single gene has been identified in members of the Eurotiales, Sordariales and Pleosporales, and in some hemiascomycetous yeast species (Elleuche \& Pöggeler, 2009a). 
In mammals, plants and algae, different isoforms of CAs are targeted to different tissues and organelles (Fabre et al., 2007; Supuran, 2008; Ynalvez et al., 2008), so it is likely that fungal CAs are also unequally distributed within the fungal cell. The first evidence for an intracellular CA comes from yeast, where CA activity was measured only inside the living cell, indicating that Saccharomyces cerevisiae does not encode a secreted CA isoform (Amoroso et al., 2005). In Sordaria macrospora, $\beta$-CAs are targeted to different subcellular compartments. CAS1 and CAS3 are cytoplasmic CAs, while CAS2 is specifically translocated into mitochondria, and the $\alpha$-CA CAS4 is predicted to be secreted (Elleuche \& Pöggeler, 2009b).

A detailed survey of CAs in the genomes of mycelial fungi demonstrated that nearly all filamentous ascomycetes investigated so far encode a single mitochondrial plantlike $\beta$-CA. Interestingly, this specific isozyme is not homologous when comparing members of the orders
Sordariales and Eurotiales, indicating that the translocation of CA into mitochondria has evoloved at least twice in the fungi (Elleuche \& Pöggeler, 2009a).

\section{CAS1 and CAS2 are involved in Sordaria macrospora fruiting body development}

A comprehensive molecular investigation of the S. macrospora $\beta$-CAs revealed that the plant-like isozymes CAS1 and CAS2 are involved in the bicarbonate-dependent regulation of fruiting body formation and maturation (Fig. 1). Surprisingly, the deletion of a single cas gene does not cause lethality, as it does in yeasts and several prokaryotes (Götz et al., 1999; Klengel et al., 2005; Kusian et al., 2002). From analysis of the phenotypes of $\Delta$ cas $1, \Delta$ cas 2 and $\Delta$ cas3 deletion strains, cas2 appears to encode the major CA in $S$. macrospora, since only the $\Delta$ cas 2 mutant is drastically impaired in vegetative growth. This

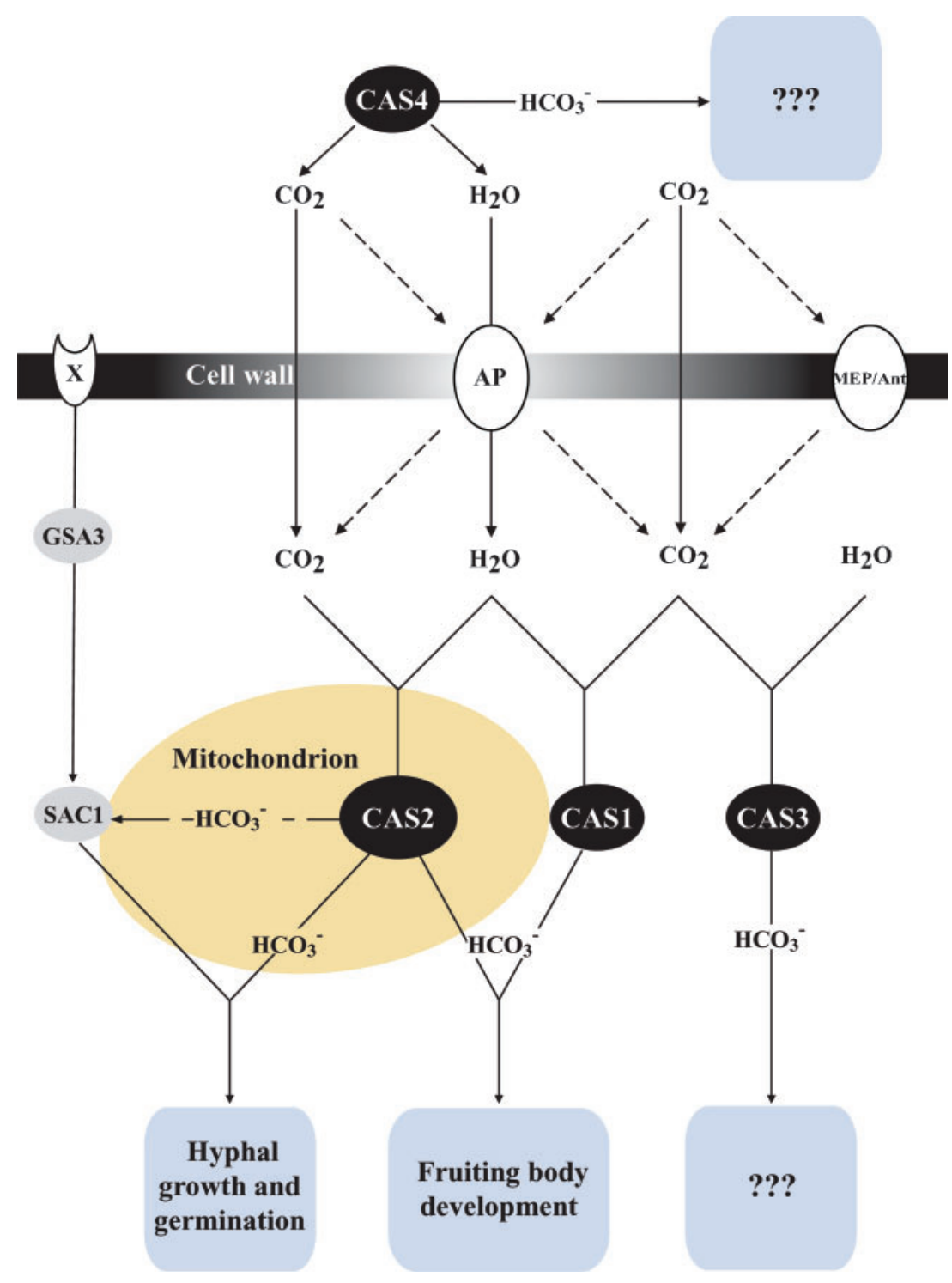

Fig. 1. Hypothetical model of bicarbonate regulation in the filamentous fungus Sordaria macrospora. $\mathrm{CO}_{2}$ is transported into the fungal cell by diffusion or putative transport systems (AP, MEP/Ant; according to Bahn \& Mühlschlegel, 2006). The figure depicts the localization and influence of various processes of $\alpha$ - and $\beta$-CAs and the adenylyl cyclase (AC) pathway in S. macrospora. The $\alpha$-CA CAS 4 is predicted to be secreted from the fungal cell, whereas CAS1 and CAS3 are cytoplasmically located and CAS2 is translocated into the mitochondria. The plant-like $\beta$-CAs CAS 1 and CAS2 play a role in fruiting body development and CAS2 is additionally involved in the regulation of hyphal growth and germination. The latter processes might be connected to the well-known G-protein/AC pathway (GSA3/SAC1). The functional roles of cablike $\beta$-CA CAS3 and $\alpha$-CAS4 are so far unknown. $X$, unknown receptor; $A P$, aquaporin; MEP/Ant, putative ammonium permease/transporter. Dashed lines indicate hypothetical signal cascades that have yet to be proven for S. macrospora. 
defect can be functionally complemented non-specifically by high $\mathrm{CO}_{2}$ concentrations, or by overexpression of cas 1 and cas3. A partial complementation of the growth defect was also achieved by providing a mixture of palmitic, stearic and myristic acid, indicating that $\mathrm{HCO}_{3}^{-}$produced by CAS2 is involved in the biosynthesis of fatty acids. In addition to the vegetative growth impairment, the germination rate of the $\Delta$ cas 2 mutant is drastically reduced compared to the wild-type, $\Delta$ cas 1 or $\Delta c a s 3$ strains. Because this defect cannot be restored by the addition of a nonspecific bicarbonate source such as elevated $\mathrm{CO}_{2}$ concentration, or by overexpression of cas 1 or cas3, S. macrospora is hypothesized to require a well-defined amount of CA activity within the mitochondria to assure efficient ascospore germination (Elleuche \& Pöggeler, 2009b). Ascospores have a depressed respiration, so their low intracellular levels of $\mathrm{HCO}_{3}^{-}$must be increased to catalyse the bicarbonate-dependent carboxylation reactions required for germination. Young germlings of the $S$. macrospora $\Delta$ cas 2 mutant are drastically impaired in growth rate and exhibit thin, highly vacuolated hyphae. In contrast to CAS2, CAS1 and CAS3 appear to provide a low level of $\mathrm{HCO}_{3}^{-}$that is sufficient only for the basal metabolic reactions of growing hyphae. Finally, the production of a mycelium from germinated $\Delta$ cas 2 spores is drastically delayed. However, when the mycelium of these colonies is consecutively transferred to fresh agar plates, the production of perithecia increases. Thus, after prolonged growth, adequate amounts of $\mathrm{CO}_{2} / \mathrm{HCO}_{3}^{-}$that are sufficient for fruiting body development accumulate in the hyphae (Elleuche \& Pöggeler, 2009b).

Comparable observations regarding the influence of CAs on developmental processes have been described in the higher plant Arabidopsis thaliana. Similar to S. macrospora, A. thaliana encodes multiple CA isoenzymes that are localized to the cytoplasm, plasma membrane and mitochondria, as well as to chloroplasts (Fabre et al., 2007; Parisi et al., 2004). The major CA of A. thaliana was shown to be the chloroplast-localized At $\beta$ CA1 (Ferreira et al., 2008). Deletion and $\mathrm{RNA}_{\mathrm{i}}$ suppression of $A t \beta \mathrm{CAl}$ result in the loss of $\geqslant 70 \%$ of total CA activity, indicating the dominant role of this specific isoform. Most of the germlings derived from $A t \beta C A 1$ mutants produce no true leaves, have a reduced root growth rate and show growth arrest after several days in ambient air conditions. All impairments can be fully restored by increased $\mathrm{CO}_{2}$ concentration in the air. However, some mutant germlings grow to maturity and are not morphologically distinguishable from the wild-type, even though their CA activity is approximately $30 \%$ of wild-type levels (Ferreira et al., 2008). The specific plastid CA activity of A. thaliana is thought to be required to mobilize energy reserves during early developmental stages, although during later stages, the activity of other CAs is sufficient for development (Ferreira et al., 2008). Similarly, in S. macrospora, the activity of the mitochondrial CAS2 enzyme seems to be especially important for the supply of $\mathrm{HCO}_{3}^{-}$during early stages of development, but during later stages, CAS1, CAS3 and CAS4 can produce sufficient bicarbonate. The interconnection and dependence of $S$. macrospora $\beta$-CAs is clearly visible in a $\Delta c a s 1 / 2$ double-deletion strain. This mutant displays an even more obvious delay in fruiting body development than the $\Delta$ cas 2 single mutant and is completely impaired in the production of mature ascospores (Elleuche \& Pöggeler, 2009b). Interestingly, deletion of the $S$. macrospora adenylyl cyclase gene sacl causes a phenotype comparable to the $\Delta$ cas 2 mutant (Kamerewerd et al., 2008). The $\Delta s a c 1$ deletion strain is impaired in vegetative growth and ascospore germination as well as in the production of fruiting bodies. While growth defects and fruiting body development are restored by the addition of cAMP to the medium, the germination defect cannot be complemented by the supply of cAMP. Furthermore, SAC1 was shown to be genetically linked to G-protein signalling (Kamerewerd et al., 2008).

By analogy to the adenylyl cyclases of Candida albicans and Crytococcus neoformans, SAC1 of S. macrospora might also be activated by bicarbonate. Because CAS2 and SAC1 seem to regulate the same processes in $S$. macrospora development, the mitochondrial CA CAS2 might provide the bicarbonate for the regulation of SAC1 activity. Interestingly, in silico analysis predicts a mitochondrial localization of SAC1 (Fig. 1) (authors' unpublished results).

\section{The Sordaria macrospora cyanase is a bicarbonate-dependent enzyme}

The CYN1 cyanase of $S$. macrospora is also regulated by bicarbonate. Cyanases catalyse the $\mathrm{HCO}_{3}^{-}$-dependent degradation of toxic cyanate in a reaction that produces $\mathrm{CO}_{2}$ and $\mathrm{NH}_{3}$ (Anderson et al., 1990). In heterotrophic bacteria and cyanobacteria, cyanases are encoded together with a CA in a cyanate-inducible operon (Guilloton et al., 1993). In E. coli, the CynT CA of the cyanase operon is important for the specific supply of $\mathrm{HCO}_{3}^{-}$for the cyanase CynS. Toxic cyanate kills cynT deletion mutants, even though a second CA is encoded in E. coli by the can gene. Under ambient air, the deletion of can is lethal. Interestingly, the lethal phenotype of a can deletion mutant can be complemented by the addition of cyanate, because this leads to the induction of the $c y n$ operon under ambient air conditions. These results indicate that the functions of $c y n S$ and $c y n T$ are strongly interconnected (Guilloton et al., 1993). The genomes of filamentous ascomycetes, but not those of hemiascomycetous yeasts, also contain a single cyanase gene, although it is not clustered with a CA gene (Elleuche \& Pöggeler, 2008; Guilloton et al., 2002). Functional characterization of the cyn1 product after heterologous expression in E. coli revealed that CYN1 catalyses the reaction of cyanate with bicarbonate to give $\mathrm{NH}_{3}$ and $\mathrm{CO}_{2}$. Transcription of the S. macrospora cyn1 gene is upregulated by the addition of cyanate to the medium, and depressed when arginine is the sole nitrogen source. 
A $\Delta c y n 1$ knockout mutant of $S$. macrospora is completely devoid of cyanase activity and shows an increased sensitivity to exogenously supplied cyanate in an arginine-depleted medium and defects in ascospore germination, but no other obvious morphological phenotypes (Elleuche \& Pöggeler, 2008). Thus, detoxification of exogenous cyanate does not seem to be the predominant function of the $S$. macrospora cyanase. From E. coli studies, cyanate appears to inhibit carbamoyl-phosphate synthetase (CPS), an enzyme responsible for the conversion of glutamine, bicarbonate and ATP to the arginine precursor carbamoyl phosphate (Anderson et al., 1973). In addition, CPS is specifically inhibited by cyanate (Guilloton \& Karst, 1987). Therefore, cyanase has been proposed to be involved in the regulation of arginine biosynthesis (Elleuche \& Pöggeler, 2008; Guilloton et al., 2002). Future analysis will focus on the functional connection between fungal CAs and cyanases, and their impact on arginine biosynthesis.

\section{Conclusion}

Recent work demonstrates that CAs have a far more extensive and fundamental role in fungal biology than previously recognized. Fungal CAs are essential for growth in ambient air because they provide bicarbonate for $\mathrm{HCO}_{3}^{-}$-dependent metabolic carboxylation reactions, such as those catalysed by pyruvate carboxylase, acetyl-CoA carboxylase and carbamoyl-phosphate synthetase. They are involved in $\mathrm{CO}_{2}$ sensing by producing bicarbonate for the activation of adenylyl cyclase. Moreover, CAs are required to establish sexual reproduction in basidiomycetes and filamentous ascomycetes. Multiple isoenzymes of filamentous ascomycetes have been found in the cytoplasm and in mitochondria, and secreted into the medium. However, in most cases very little is known about the exact physiological role for each of these isoforms. Genome sequencing projects have identified multiple CAs in several fungal species. The future challenge for fungal biologists will be to determine the biological functions of these enzymes.

\section{References}

Aguilera, J., Petit, T., de Winde, J. H. \& Pronk, J. T. (2005a). Physiological and genome-wide transcriptional responses of Saccharomyces cerevisiae to high carbon dioxide concentrations. FEMS Yeast Res 5, 579-593.

Aguilera, J., Van Dijken, J. P., De Winde, J. H. \& Pronk, J. T. (2005b). Carbonic anhydrase (Nce103p): an essential biosynthetic enzyme for growth of Saccharomyces cerevisiae at atmospheric carbon dioxide pressure. Biochem J 391, 311-316.

Amoroso, G., Morell-Avrahov, L., Müller, D., Klug, K. \& Sültemeyer, D. (2005). The gene NCE103 (YNL036w) from Saccharomyces cerevisiae encodes a functional carbonic anhydrase and its transcription is regulated by the concentration of inorganic carbon in the medium. Mol Microbiol 56, 549-558.

Anderson, P. M., Carlson, J. D., Rosenthal, G. A. \& Meister, A. (1973). Effect of potassium cyanate on the catalytic activities of carbamyl phosphate synthetase. Biochem Biophys Res Commun 55, 246-252.
Anderson, P. M., Sung, Y. C. \& Fuchs, J. A. (1990). The cyanase operon and cyanate metabolism. FEMS Microbiol Rev 7, 247-252.

Bahn, Y. S. \& Mühlschlegel, F. A. (2006). $\mathrm{CO}_{2}$ sensing in fungi and beyond. Curr Opin Microbiol 9, 572-578.

Bahn, Y. S., Cox, G. M., Perfect, J. R. \& Heitman, J. (2005). Carbonic anhydrase and $\mathrm{CO}_{2}$ sensing during Cryptococcus neoformans growth, differentiation, and virulence. Curr Biol 15, 2013-2020.

Bahn, Y. S., Xue, C., Idnurm, A., Rutherford, J. C., Heitman, J. \& Cardenas, M. E. (2007). Sensing the environment: lessons from fungi. Nat Rev Microbiol 5, 57-69.

Chen, Y., Cann, M. J., Litvin, T. N., lourgenko, V., Sinclair, M. L., Levin, L. R. \& Buck, J. (2000). Soluble adenylyl cyclase as an evolutionarily conserved bicarbonate sensor. Science 289, 625-628.

Clark, D., Rowlett, R. S., Coleman, J. R. \& Klessig, D. F. (2004). Complementation of the yeast deletion mutant DeltaNCE103 by members of the beta class of carbonic anhydrases is dependent on carbonic anhydrase activity rather than on antioxidant activity. Biochem J 379, 609-615.

Cleves, A. E., Cooper, D. N., Barondes, S. H. \& Kelly, R. B. (1996). A new pathway for protein export in Saccharomyces cerevisiae. J Cell Biol 133, 1017-1026.

Cronk, J. D., Endrizzi, J. A., Cronk, M. R., O’Neill, J. W. \& Zhang, K. Y. (2001). Crystal structure of $E$. coli beta-carbonic anhydrase, an enzyme with an unusual pH-dependent activity. Protein Sci 10, 911922.

Elleuche, S. \& Pöggeler, S. (2008). A cyanase is transcriptionally regulated by arginine and involved in cyanate decomposition in Sordaria macrospora. Fungal Genet Biol 45, 1458-1469.

Elleuche, S. \& Pöggeler, S. (2009a). Evolution of carbonic anhydrases in fungi. Curr Genet 55, 211-222.

Elleuche, S. \& Pöggeler, S. (2009b). Beta-carbonic anhydrases play a role in fruiting body development and ascospore germination in the filamentous fungus Sordaria macrospora. PLoS One 4, e5177.

Fabre, N., Reiter, I. M., Becuwe-Linka, N., Genty, B. \& Rumeau, D. (2007). Characterization and expression analysis of genes encoding alpha and beta carbonic anhydrases in Arabidopsis. Plant Cell Environ 30, 617-629.

Ferreira, F. J., Guo, C. \& Coleman, J. R. (2008). Reduction of plastidlocalized carbonic anhydrase activity results in reduced Arabidopsis seedling survivorship. Plant Physiol 147, 585-594.

Götz, R., Gnann, A. \& Zimmermann, F. K. (1999). Deletion of the carbonic anhydrase-like gene NCE103 of the yeast Saccharomyces cerevisiae causes an oxygen-sensitive growth defect. Yeast 15, 855-864.

Guilloton, M. \& Karst, F. (1987). Cyanate specifically inhibits arginine biosynthesis in Escherichia coli K12: a case of by-product inhibition? J Gen Microbiol 133, 655-665.

Guilloton, M. B., Lamblin, A. F., Kozliak, E. I., Gerami-Nejad, M., Tu, C., Silverman, D., Anderson, P. M. \& Fuchs, J. A. (1993). A physiological role for cyanate-induced carbonic anhydrase in Escherichia coli. J Bacteriol 175, 1443-1451.

Guilloton, M., Espie, G. S. \& Anderson, P. M. (2002). What is the role of cyanase in plants? In Reviews in Plant Biochemistry and Biotechnology, pp. 57-79. Edited by A. Goyal, S. L. Metha \& M. L. Lodha.

Innocenti, A., Leewattanapasuk, W., Muhlschlegel, F. A., Mastrolorenzo, A. \& Supuran, C. T. (2009). Carbonic anhydrase inhibitors. Inhibition of the beta-class enzyme from the pathogenic yeast Candida glabrata with anions. Bioorg Med Chem Lett 19, 48024805.

Jones, N. L. (2008). An obsession with $\mathrm{CO}_{2}$. Appl Physiol Nutr Metab 33, 641-650. 
Kamerewerd, J., Jansson, M., Nowrousian, M., Pöggeler, S. \& Kück, U. (2008). Three alpha-subunits of heterotrimeric G proteins and an adenylyl cyclase have distinct roles in fruiting body development in the homothallic fungus Sordaria macrospora. Genetics 180, 191-206.

Kimber, M. S. \& Pai, E. F. (2000). The active site architecture of Pisum sativum beta-carbonic anhydrase is a mirror image of that of alphacarbonic anhydrases. EMBO J 19, 1407-1418.

Klengel, T., Liang, W. J., Chaloupka, J., Ruoff, C., Schröppel, K., Naglik, J. R., Eckert, S. E., Mogensen, E. G., Haynes, K. \& other authors (2005). Fungal adenylyl cyclase integrates $\mathrm{CO}_{2}$ sensing with cAMP signaling and virulence. Curr Biol 15, 2021-2026.

Kück, U., Pöggeler, S., Nowrousian, M., Nolting, N. \& Engh, I. (2009). Sordaria macrospora, a model system for fungal development. In The Mycota XV, pp. 17-39. Edited by T. Anke \& D. Weber. Berlin, Heidelberg: Springer.

Kusian, B., Sültemeyer, D. \& Bowien, B. (2002). Carbonic anhydrase is essential for growth of Ralstonia eutropha at ambient $\mathrm{CO}_{2}$ concentrations. J Bacteriol 184, 5018-5026.

Li, W., Zhou, P. P., Jia, L. P., Yu, L. J., Li, X. L. \& Zhu, M. (2009). Limestone dissolution induced by fungal mycelia, acidic materials, and carbonic anhydrase from fungi. Mycopathologia 167, 37-46.

Mogensen, E. G. \& Mühlschlegel, F. A. (2008). $\mathrm{CO}_{2}$ sensing and virulence of Candida albicans. In The Mycota VI, pp. 83-94. Edited by A. A. Brackhage \& P. F. Zipfel. Berlin, Heidelberg: Springer.

Mogensen, E. G., Janbon, G., Chaloupka, J., Steegborn, C., Fu, M. S., Moyrand, F., Klengel, T., Pearson, D. S., Geeves, M. A. \& other authors (2006). Cryptococcus neoformans senses $\mathrm{CO}_{2}$ through the carbonic anhydrase Can2 and the adenylyl cyclase Cac1. Eukaryot Cell 5, 103-111.

Nishida, H., Beppu, T. \& Ueda, K. (2009). Symbiobacterium lost carbonic anhydrase in the course of evolution. J Mol Evol 68, 90-96.

Nowrousian, M., Würtz, C., Pöggeler, S. \& Kück, U. (2004). Comparative sequence analysis of Sordaria macrospora and Neurospora crassa as a means to improve genome annotation. Fungal Genet Biol 41, 285-292.
Parisi, G., Perales, M., Fornasari, M. S., Colaneri, A., GonzálezSchain, N., Gómez-Casati, D., Zimmermann, S., Brennicke, A., Araya, A. \& other authors (2004). Gamma carbonic anhydrases in plant mitochondria. Plant Mol Biol 55, 193-207.

Pöggeler, S., Nowrousian, M. \& Kück, U. (2006). Fruiting body development in ascomycetes. In The Mycota I, pp. 325-355. Edited by U. Kües \& R. Fischer. Berlin \& Heidelberg: Springer.

Sawaya, M. R., Cannon, G. C., Heinhorst, S., Tanaka, S., Williams, E. B., Yeates, T. O. \& Kerfeld, C. A. (2006). The structure of betacarbonic anhydrase from the carboxysomal shell reveals a distinct subclass with one active site for the price of two. J Biol Chem 281, 7546-7555.

Schlicker, C., Hall, R. A., Vullo, D., Middelhaufe, S., Gertz, M., Supuran, C. T., Mühlschlegel, F. A. \& Steegborn, C. (2009). Structure and inhibition of the $\mathrm{CO}_{2}$-sensing carbonic anhydrase Can2 from the pathogenic fungus Cryptococcus neoformans. J Mol Biol 385, 12071220.

Slaymaker, D. H., Navarre, D. A., Clark, D., del Pozo, O., Martin, G. B. \& Klessig, D. F. (2002). The tobacco salicylic acid-binding protein 3 (SABP3) is the chloroplast carbonic anhydrase, which exhibits antioxidant activity and plays a role in the hypersensitive defense response. Proc Natl Acad Sci U S A 99, 11640-11645.

Supuran, C. T. (2008). Carbonic anhydrases - an overview. Curr Pharm Des 14, 603-614.

Tripp, B. C., Smith, K. \& Ferry, J. G. (2001). Carbonic anhydrase: new insights for an ancient enzyme. J Biol Chem 276, 48615-48618.

Wistrand, P. J. (1981). The importance of carbonic anhydrase B and C for the unloading of $\mathrm{CO}_{2}$ by the human erythrocyte. Acta Physiol Scand 113, 417-426.

Xu, Y., Feng, L., Jeffrey, P. D., Shi, Y. \& Morel, F. M. (2008). Structure and metal exchange in the cadmium carbonic anhydrase of marine diatoms. Nature 452, 56-61.

Ynalvez, R. A., Xiao, Y., Ward, A. S., Cunnusamy, K. \& Moroney, J. V. (2008). Identification and characterization of two closely related betacarbonic anhydrases from Chlamydomonas reinhardtii. Physiol Plant $133,15-26$. 